\title{
LA REGISTRAZIONE DEI TERREMOTI A PAVIA
}

\author{
G. Pannocchia
}

Trasferito nell'Osservatorio Geofisico di Pavia chiesi ed ottenni dalla sede centrale dell'Istituto Nazionale di Geofisica di poter aygriungere ai due sismografi Wiechert, già funzionanti ottimamente, due sismografi tipo Galitzin. Voglio augurarmi che possa essere presto completata la terna di questi apparecchi e che quindi questa stazione possa allinearsi alle altre stazioni Italiane ed estere nella stesura dei bollettini microsismici, oltre che in quella, già in atto, dei bollettini sismici. I microsismi si manifestano a Pavia in proporzioni piuttosto eccezionali e sarebbe ottima cosa poterli seguire dalla registrazione nelle tre componenti classiche. Attualmente si registrano quasi tutti i terremoti.

Gli apparecchi in funzione sono un sismografo astatico Wiechert da $80 \mathrm{~K}_{\mathrm{g}}$ con un periodo proprio di $3^{\mathrm{s}}, 1$ : un sismografo astatico Wiechert da $200 \mathrm{kr}$ con un periodo proprio di $3^{*}, 34$ per la componente N-S, e di $3^{*}, 09$ per la componente $\mathrm{E}-\mathrm{W}$; un sismografo Galitzin componente N.S (registrazione su carta fotografica) con un periodo proprio di $17 \times 2$; un sismografo astatieo Galitzin-Pannocchia (1) comp. verticale con un periodo proprio di $17^{\mathrm{s}}, 2$.

Per la stazione sismica di Pavia è stata realizzata una carta delle isodistanti ed isoazimutali (con centro Pavia) analoga a quella già in uso a Roma (2); ed inoltre $\dot{e}$ stata calcolata la formula valevole per la determinazione della "Magnitudo " dei terremoti dai dati sperimentali di Pavia.

Per questo scopo si è fatto uso della formula $\left({ }^{3}\right)$

$$
m=\log A+y \log \Delta+x
$$

per la quale $\mathrm{i}$ valori delle incognite sono stati determinati con il metodo dei minimi quadrati, partendo dai dati tratti da un gruppo di 62 terremoti per i quali era già stata calcolata la "Magnitudo » da Pasadena: essa ha quindi preso per Pavia l'aspetto $\left({ }^{4}\right)$ :

$$
m=\log . .4+2.641 \log \Delta+0339
$$


( $A$ ampiezza in micron dello spostamento reale massimo del suolo per un periodo di circa 20": $\rfloor$ distanza geocentrica dell'epicentro dalla stazione).

L'uso della formula ha mostrato che i risultati per la " magnitudo" sono concordi a quelli calcolati in altre stazioni; tuttavia non se ne dà notizia nei bollettini per irregolarità nella continuita di funzionamento degli apparecchi a registrazione fotografica. La determinazione della magnitudo viene facilitata dall'uso del grafico allegato (fig. $l$.

Da quando sono a Pavia avendo riscontrato nelle interpretazioni originali dei sismowrammi notevoli differenze sia nei tempi di registrazione dai confronti con i bollettini internazionali editi a Straslurgo, sia nella determinazione delle distanze che spesso risulta errata, pur non riconoscendomi particolari capacita di lettura ed interpretazione dei sismogrammi, ho tuttavia ritenuto cosa utile esaminare il fatto più accuratamente per cercare di trovare la causa di tali discordanze.

A questo scopo ho preso in esame dai registri dei terremoti degli anni 1951-1952-1953 tutti quei terremoti per i quali erano state da me indicate le onde $P$ ed $S$, o, in qualche caso, solo le $P$. Per ognuno di essi dai bollettini mensili dell'U.G.G.I., editi a Strasluurgo, ho tratto le coordinate geografiche, l'ora orignine, la profondità, quando indicata, assegnate al terremoto dall'L'S.C.G.S. o, in difetto. dalla B.C.I.S.

Quindi usando tali dati ho calcolato per ciascuno di essi la distanza geocentrica da Pavia, con le note formule, ed i tempi di tragitto sia per le $P$ che per le $S$ fora di registrazione - ora origine indicata nel bollettino). Successivamente ho confrontato tali tempi di tragitto con quelli indicati rispettivamente nelle tavole di P. Caloi ("i. di H. Jeffreys, di G. J. Brunner a seconda della distanza calcolata e della profondità indicata.

Da questo confronto $\dot{e}$ risultato in modo evidente che le $P$ griungono alla stazione di Pavia, nella stragrande maggioranza dei casi, con un ritardo sensibile rispetto alle tabelle di marcia prima indicate. In moltissimi casi anzi, onde rendere tale ritardo di entità ragionevole, ho dovuto (quando possibile) confrontare il tempo di tragitto trovato con quello indicato in corrispondenza alla colonna "surface " della tabella di Jeffreys: o in caso di terremoti profondi, con i tempi di tragitto relativi a profondita inferiori (notevolmente) a quelle indicate. Pochi sono i casi nei quali si sono riscontrati anticipi: in questi casi sia che si tratti di terremoti per i quali è indicata la profondità, 


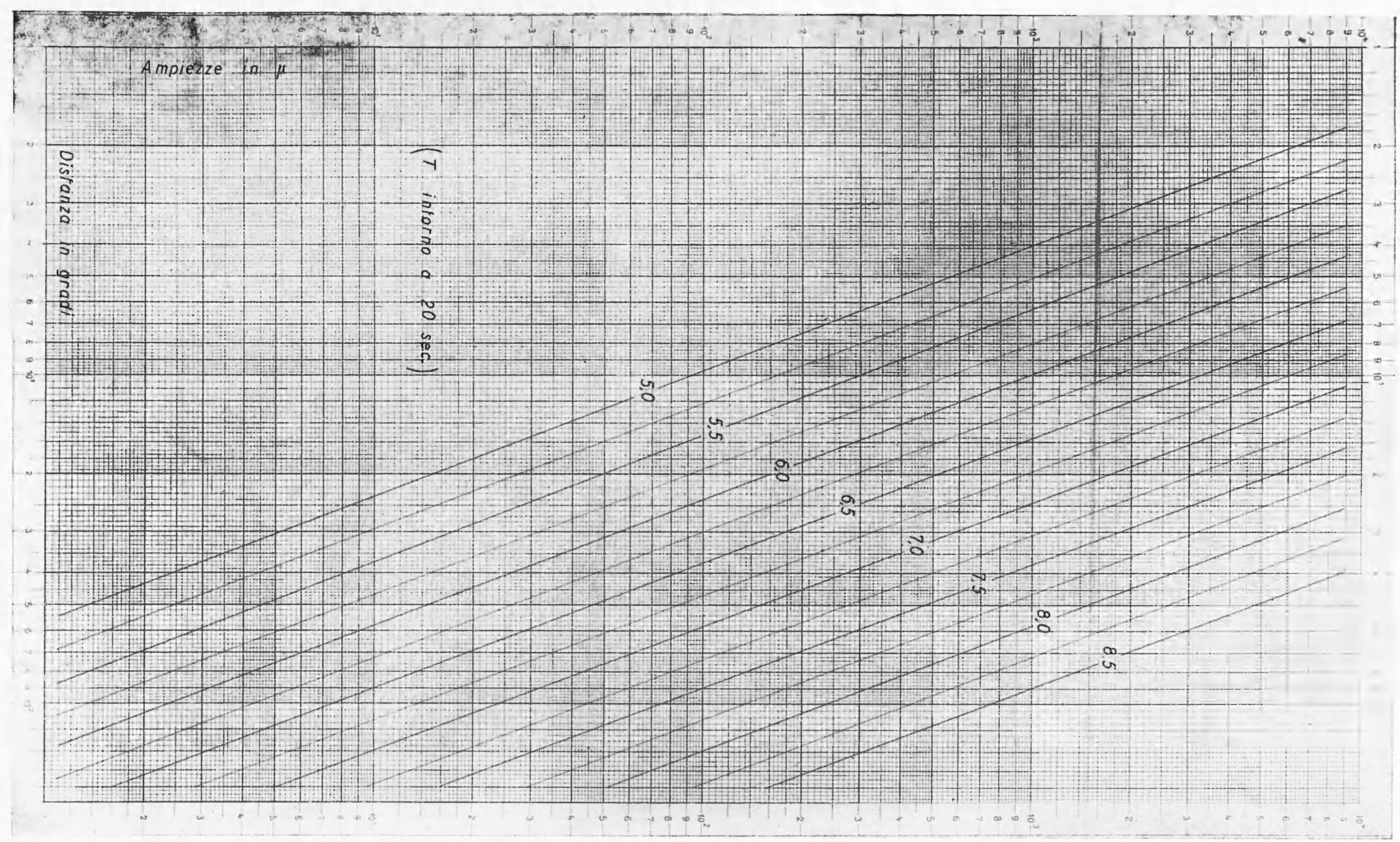


sia che si tratti di terremoti per i quali non è indicata la profondità, l'anticipo può essere spiegato come derivante dall'essere il terremoto a profondità maggiore della normale o maggiore della indicata.

Oltre la indicazione qualitativa non mi è sembrato di poter de. durre dai dati ottenuti una indicazione quantitativa poiché bisogna tener conto che le determinazioni epicentrali dell'U.S.C.G.S. o della B.C.I.S., pur essendo precise, non sono tali da render certa l'ora origine oltre un certo limite: tale limite può anche essere dell'ordine di grandezza del ritardo medio riscontrato da me. Rimane tuttavia valida l'osservazione qualitativa: la prevalenza dei ritardi nei tempi di arrivo delle $P$ ed $S$ e queste ultime, in un numero notevole di casi (49) non si sono potute considerare come tali dal confronto dei tempi di tragitto indicati dalle tabelle. Le seguenti tavole riassuntive generali mostrano chiaramente l'andamento del fenomeno.

Totale dei terremoti presi in esame 242

Terremoti scartati per ritardi o anticipi $>10^{\text {s }}$ per le $P$ ed $S, 22$

\begin{tabular}{|c|c|c|c|c|c|c|c|}
\hline Tav. I & \multicolumn{7}{|c|}{ Onde $P$} \\
\hline \multirow[b]{2}{*}{ Rit. $>10^{\circ} \mathrm{co}_{\mathrm{a}}$} & \multirow[b]{2}{*}{ Rit. $>5^{*}$} & \multirow[b]{2}{*}{ Rit. $<5^{s}$} & \multicolumn{3}{|c|}{ Coincidenti } & \multirow[b]{2}{*}{ Ant. $>5$} & \multirow[b]{2}{*}{ Ant. $<5^{n}$} \\
\hline & & & $\mathrm{h}>33$ & $0<\mathrm{h}<33$ & $\begin{aligned} \mathrm{h}< & =33 \\
& =0\end{aligned}$ & & \\
\hline 9 & 33 & 110 & 10 & 14 & 14 & 3 & 27 \\
\hline Tot. & t. & 152 & coinc. & & 38 & ant. & 30 \\
\hline
\end{tabular}

Per i 38 coincidenti c'è da precisare che di essi 10 erano più profondi di $33 \mathrm{~km}$ e che per un opportuno valore della profondità sono stati considerati coincidenti, mentre presentavano ritardo per la pro-

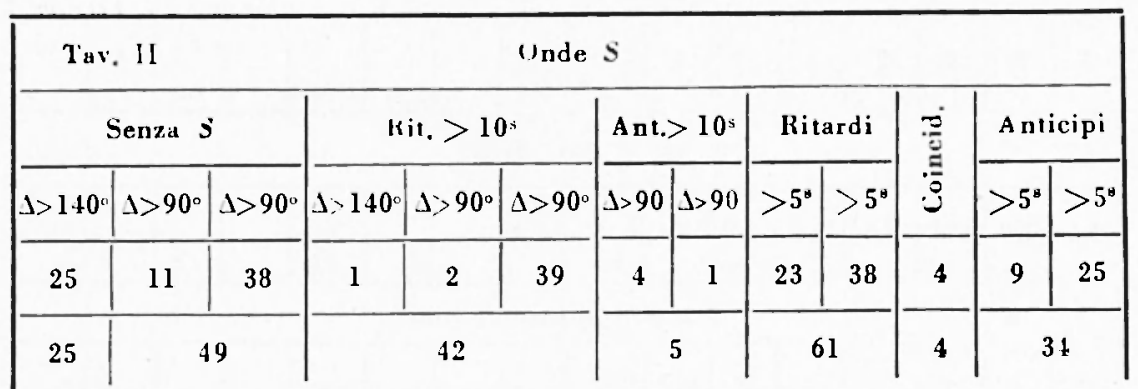

Tut. Kit. 103 lut, Ant 39 
fondita indicata; 11 presentavano ritardo per profondità di $33 \mathrm{~km}$ ed anticipo per profonditì "surface "; gli altri 14 presentavano realmente coincidenza o per profondità di $33 \mathrm{~km}$ o di $0 \mathrm{~km}$ ( ( surface )).

Oltre alle precedenti tavole mi è sembrato opportuno riassumere le tavole non riportate qui nelle seguenti (valori medi di $\Delta t$. e $0^{\mathrm{s}}<\Delta t$ $<5^{\mathrm{s}}$ :

\begin{tabular}{|c|c|c|c|c|c|c|c|c|c|c|c|c|c|c|c|}
\hline \multicolumn{4}{|c|}{ rav. III } & \multicolumn{4}{|c|}{$0^{0}<u<450$} & \multicolumn{4}{|c|}{$\left.25^{\circ}<\right\lrcorner<140^{\circ}$} & & & & \\
\hline \multicolumn{4}{|c|}{$\mathrm{h}=0 \mathrm{Km}$} & \multicolumn{4}{|c|}{$\mathrm{h}=33 \mathrm{~K} \cdot \mathrm{m}$} & \multicolumn{4}{|c|}{$h=63,4 k m$} & \multicolumn{4}{|c|}{$h_{1}>63.4 \mathrm{Km}$} \\
\hline \multicolumn{2}{|c|}{ Rit in sec. } & \multicolumn{2}{|c|}{ Ant. in sec. } & \multicolumn{2}{|c|}{ Rit. in ser. } & \multicolumn{2}{|c|}{ Int in sec: } & \multicolumn{2}{|c|}{ Rit in sec. } & \multicolumn{2}{|c|}{ Int in sec. } & \multicolumn{2}{|c|}{ kit in sec. } & \multicolumn{2}{|c|}{ Ant in sec. } \\
\hline$P$ & $S$ & $\mathbf{P}$ & S & $\mathbf{P}$ & $s$ & $p$ & $s$ & $P$ & $s$ & $r$ & S & $\mu$ & $\mathrm{s}$ & $\mathbf{P}$ & $s$ \\
\hline 2,5 & 2,2 & 1,4 & 2,6 & 2,3 & 2,3 & 0,8 & 3,3 & 1,4 & 3,3 & 1,8 & & 2,6 & & & $(6,2)$ \\
\hline \multicolumn{16}{|c|}{ 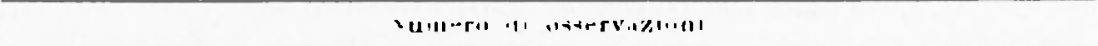 } \\
\hline 31 & 14 & 10 & 3 & 20 & : & 6 & 6 & 1 & 2 & 1 & & 3 & & & 1 \\
\hline
\end{tabular}

\begin{tabular}{|c|c|c|c|c|c|c|c|c|c|c|c|c|c|c|c|}
\hline \multicolumn{6}{|c|}{ Tav. iv } & \multicolumn{10}{|c|}{$450<a<1120,5$} \\
\hline \multicolumn{16}{|c|}{ 5o $<\unlhd<400$} \\
\hline \multicolumn{4}{|c|}{$\mathrm{h}=0 \mathrm{Km}$} & \multicolumn{4}{|c|}{$\mathbf{h}=33 \mathbf{K} m$} & \multicolumn{4}{|c|}{$\mathrm{h}=63,4 \mathrm{~km}$} & \multicolumn{4}{|c|}{$\mathrm{h}>63,4 \mathrm{~km}$} \\
\hline \multicolumn{2}{|c|}{ Rit. in sec. } & \multicolumn{2}{|c|}{ Ant in sec. } & \multicolumn{2}{|c|}{ Rit. in sec. } & \multicolumn{2}{|c|}{ Ant in sec. } & \multicolumn{2}{|c|}{ Kit. in sec. } & \multicolumn{2}{|c|}{ Ant. in sec. } & \multicolumn{2}{|c|}{ Rit. in sec. } & \multicolumn{2}{|c|}{ Ant in sec. } \\
\hline$r$ & $\bar{s}$ & $\mu$ & $s$ & $r$ & $s$ & $r$ & 5 & ' & $s$ & 11 & 5 & $P$ & $s$ & $f^{\prime}$ & $\therefore$ \\
\hline 4,5 & 2,1 & 0,7 & 3,8 & 3,5 & & 4,0 & & 3,7 & & & & & & 0,5 & \\
\hline \multicolumn{16}{|c|}{ Numeru di osservazım } \\
\hline 5 & 3 & 4 & 2 & 1 & & 1 & & 1 & & & & & & 1 & \\
\hline \multicolumn{16}{|c|}{$45^{\circ}<\Delta<1000^{\circ}$} \\
\hline 1,5 & 3,9 & 0,9 & 2,6 & 3,0 & 2,9 & 3,4 & & 4,0 & & & 3,2 & & & & \\
\hline \multicolumn{16}{|c|}{ Numero di oservilzioni } \\
\hline 12 & 4 & 3 & 3 & 5 & 2 & 1 & & 2 & & & 1 & & & & \\
\hline
\end{tabular}




\begin{tabular}{|c|c|c|c|c|c|c|c|c|c|c|c|c|c|c|c|}
\hline \multicolumn{5}{|c|}{ Yav, V } & \multicolumn{7}{|c|}{$1120,5<\alpha<3250$} & & & & \\
\hline \multicolumn{4}{|c|}{$10^{3}<د<25^{\circ}$} & \multicolumn{12}{|c|}{$45^{\circ}<1<90^{\circ}$} \\
\hline \multicolumn{4}{|c|}{$\mathrm{h}=0 \mathrm{~km}$} & \multicolumn{4}{|c|}{$11=0 K_{111}$} & \multicolumn{4}{|c|}{$\mathrm{h}=33 \mathrm{Km}$} & \multicolumn{4}{|c|}{$h=63, t$} \\
\hline \multicolumn{2}{|c|}{ Rat. in ser. } & \multicolumn{2}{|c|}{ Int. in sec. } & \multicolumn{2}{|c|}{ Rit. in sec. } & \multicolumn{2}{|c|}{ Int in sec. } & \multicolumn{2}{|c|}{ Rit. in sec. } & \multicolumn{2}{|c|}{ Ant.in ser. } & \multicolumn{2}{|c|}{ Rit. in sec. } & \multicolumn{2}{|c|}{ Int in ser } \\
\hline I' & 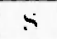 & $\mathbf{P}$ & $s$ & $\mathbf{P}$ & $\mathrm{S}$ & $\mathbf{P}$ & $\therefore$ & $p$ & $\$$ & $p$ & $S$ & 1 & $\therefore$ & $p$ & - \\
\hline 2,5 & 3.7 & 3,7 & 3,3 & 1.8 & & 1.3 & & 3,5 & & & & 4,5 & & & \\
\hline \multicolumn{16}{|c|}{ Numero di osservaziuni } \\
\hline 17 & 5 & 5 & 5 & 4 & & 1 & & 1 & & & & 1 & & & \\
\hline
\end{tabular}

\begin{tabular}{|c|c|c|c|c|c|c|c|c|c|c|c|c|c|c|}
\hline \multicolumn{4}{|c|}{ Tax. $v 1$} & \multicolumn{6}{|c|}{$225^{\circ}<\mathrm{a}<360^{\circ}$} & \multicolumn{5}{|c|}{$400<1<1000$} \\
\hline \multicolumn{4}{|c|}{$\mathrm{h}=0 \mathrm{Km}$} & \multicolumn{4}{|c|}{$h=33 \mathrm{~K}$} & \multicolumn{4}{|c|}{$n=63,4 k \ldots$} & \multirow{2}{*}{\multicolumn{3}{|c|}{$\begin{array}{c}\mathrm{h}>63, \mathrm{t} \\
\text { Rit. in sec. } \\
\text { Ant. in sec }\end{array}$}} \\
\hline \multicolumn{2}{|c|}{ Rit. in sec. } & \multicolumn{2}{|c|}{ Ant in ser. } & \multicolumn{2}{|c|}{ Rit. in sec. } & \multicolumn{2}{|c|}{ fut. in sec. } & \multicolumn{2}{|c|}{ Rit. in ser. } & \multicolumn{2}{|c|}{ Ant. in spe. } & & & \\
\hline $\mathbf{P}$ & $s$ & $p$ & $S$ & $P$ & $\mathrm{~S}$ & $P$ & $S$ & $\mathbf{p}$ & $s$ & $P$ & 5 & 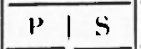 & $r^{\prime}$ & $\therefore$ \\
\hline 2,3 & 2,9 & 1,3 & 1.0 & 3,0 & 2,3 & 1,9 & 1,1 & 2,6 & & 2,0 & & & & \\
\hline & & & & & & ume & di & usser & sain, & & & & & \\
\hline 12 & 4 & 5 & 3 & 6 & 1 & 2 & 1 & 2 & & 1 & & & & \\
\hline
\end{tabular}

Le medie per i ritardi delle $P$ ed $S$ dalle precedenti tavole usando i valori medi relativi al maggior numero di osservazioni danno nei quattro settori di provenienza prescelti:

$$
0^{\prime \prime}<x<45^{\circ} \text { Rit. }\left\{\begin{array}{l}
P 2^{*}, 4 \\
S 2^{*}, 25
\end{array} \quad I\right. \text { settore }
$$

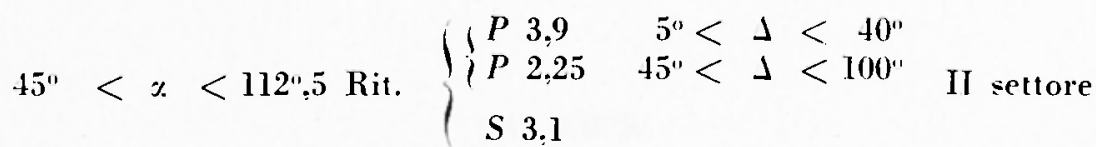

$112^{\circ}, 5<\%<225^{\prime \prime} \quad$ Rit. $\left\{\begin{array}{lll}P & 2,5 & 10^{\prime \prime}<J<25^{\prime \prime} \\ P & 2,0 & 45^{\prime \prime}<J<90^{\prime \prime} \\ S & 3,7\end{array}\right.$ III settore $225^{\circ}<x<360^{\circ} \quad$ Rit. $\left\{\begin{array}{l}P 2,6 \\ S 2,6\end{array} \quad\right.$ IV settore 
Da queste medie sembrerebhe di poter concludere che le $S$ abbiano un maggiore ritardo delle $P$, tranne che nel primo settore; che le $P$ abbiano un maggiore ritardo per terremoti entro i $40^{\prime \prime}$ di distanza nei due settori II e III. inferiore per distanze maggiori di $45^{\circ}$. Sembra ragionevole concludere inoltre che $i$ ritardi riscontrati siano dovuti all'effetto della coltre padana, in cui le velocità di propagazione, evidentemente, per le $P$ e per le $S$ vengono ad essere ridotte. Ciò è stato messo in rilievo, per gli strati più superficiali e nei dintorni di Pavia, da G. Aliverti ed L. Solaini $\left({ }^{6}\right)$; le mie conclusioni riguarderebbero tutto lo spessore del sedimento padano del quale per altro, con i dati a disposizione, per le ragioni già esposte, non mi è sembrato di poter valutare né lo spessore, né la velocità di propagazione per le singole onde.

Il notevole numero di casi in cui le $S$ non collimano con le tav. d'uso normale, ben $42+49+5=96$ su 242 terremoti, giustificano le discordanze riscontrate di frequente nella determinazione delle distanze, mentre il gran numero di ritardi nelle $P$ osservati giustificano le divergenze riscontrate rispetto ai bollettini per i tempi di registrazione in Pavia.

Istituto Nazionale di Geofisica - Osserv. di Pavia - Marzo 1955.

\section{RIASSUNTO}

Dopo alcune notizie generali sulla stazione sismica di Pavia, si mette in rilievo come in questa stazione si riscontri un sensibile ritardo nei tempi di arrivo delle onde $\mathrm{P}$ ed $\mathrm{S}$; tale ritardo si attribuisce ad una minore velocità di propagazione delle dette onde attraverso la coltre padana.

\section{$S U M M A R Y$}

Some general information on the seismic station of Pavia are given. We point out that in this station an appreciable time delay in the arrival of the $\mathrm{P}$ and $\mathrm{S}$ waves is observed; we attribute this delay to a less velocity of propagation of the seismic uaves through the superficial Padan layers. 


\section{BIBLIOGRAFIA}

(1) G. Panxoccina, Sismografo verticule a 20 di periodo proprio. La Ricerca Srientifica, anno $X$, n. 3, pag. 119.

(-) A. Lo Strno, La registrazione e lo studio dei fenomeni sismici nell'Istituto Nazionale di Geofisica del C.N.R. La Ricerca Scientifica, anno XI, n. 19, ott. 1940.

(3) D. D) Fitip'o - L. Manctilu, La Magnitudo dei terremoti e la sua determinazione nella stazione sismica di Roma. Annali di Geofisiea, vol. II, n. 4, pag. 486.

(t) A. F. Beblsa, Ringrazio qui il dr. A. F. Bedina per averni messo a disposizione la sua tesi di laurea, da cui sono stati tratti i dati riportati nel testo relativamente alla "Magnitudo " e la fig. 1.

(.1) P'. Catol. Tempi di tragitto per terremoti ad origine vicina. La Ricerca Scientifica. anno $X$, n. 5 , pag. 388 .

(ii) G. Aliverti - I. Solasi. Sulla velocitì di propagazione delle onde sismiche su brevi percorsi superficiali. Rivista di Geofisica Applieata, anno XI, n. 1, pag. 3. 